\title{
Critical flow in open channels: Numerical solution using the Newton-Raphson method for Android 4.0 application
}

\section{Flujo crítico en canales abiertos, solución numérica mediante el método de Newton-Raphson para aplicación Android 4.0}

\author{
Édgar Orlando Ladino Moreno ${ }^{1}$, César Augusto García-Ubaque ${ }^{2}{ }^{(D,}$ \\ María Camila García-Vaca ${ }^{3}$ (i)
}

Cómo citar: Ladino-Moreno, E.O., García-Ubaque, C.A. y García-Vaca, M.C. (2020). Critical flow in open channels: Numerical solution using the Newton-Raphson method for Android 4.0 application. Tecnura, 24(63) 88-103. DOI: https://doi.org/10.14483/22487638.16196

Fecha de de recepción: 10 de junio de 2019

\section{ABSTRACT}

Context: In this article we present an algorithm under JavaScript source code for critical flow equation solution.

Method: An object-oriented programming language was implemented for Android 4.0 or higher systems based on iterative and incremental processes (Agile development). The numerical method of Newton-Raphson was used to determine the critical depth of seven hydraulic sections (rectangular, trapezoidal, asymmetric trapezoidal, triangular, asymmetric triangular, parabolic and circular). A potential function was obtained to establish the seed value in iterative process, in order to accelerate and guarantee the convergence level for each
Fecha de de aceptación: 18 de octubre de 2019

section. This value is directly associated with hydraulic problem pre-established conditions.

Results: The application calculates: critical depth, critical speed, hydraulic area, specific energy, wet perimeter and mirror. The results calculated by the application were validated against Excel analysis tool (Goal Seek) results and Hcanales ${ }^{\circledR}$ software developed by Máximo Villón Béjar Engineer. Finally, the application is available for free in Google Play Store, with the name "Critical Flow in Channels. Newton Raphson Solution".

Conclusions: It is possible to develop easily accessible applications that meet the technical conditions required for the resolution of engineering-related situations.

Keywords: application software, educational software, water flow, fluid dynamics, JavaScript code.

1 Civil Engineer, Universidad Militar Nueva Granada. Topographic Engineer, Universidad Distrital Francisco José de Caldas, Colombia. MsC Civil Engineering, Escuela Colombiana de Ingeniería, Colombia. Associated Profesor asistente, Universidad Distrital Francisco José de Caldas. Contacto: edgar.ladino@unimilitar.edu.co. ORCID: https://orcid.org/0000-0002-7770-452X

2 Civil Engineer, Universidad Nacional de Colombia, Colombia. MsC Civil Engineering, Universidad de los Andes, Colombia. PhD in Engineering, Universidad de los Andes, Colombia. Titular Professor Universidad Distrital Francisco José de Caldas, Colombia. Contacto: cagarciau@udistrital.edu.co ORCID: https://orcid.org/0000-0002-6959-6610

3 Chemical Engineer, Universidad de los Andes, Colombia. MsC Chemical Engineering, Universidad de los Andes, Colombia. Professor Universidad Católica de Colombia, Colombia. Contacto: mcgarciav@ucatolica.edu.co

ORCID: https://orcid.org/0000-0002-1382-7387 


\section{RESUMEN}

Contexto: En este artículo se presenta el desarrollo de un algoritmo bajo código fuente JavaScript para la solución de la ecuación de flujo crítico.

Método: Se implementó un lenguaje de programación orientado a objetos para sistemas Android 4.0 o superiores, a partir de procesos iterativos e incrementales (desarrollo ágil). Se utilizó el método numérico de Newton-Raphson para determinar la profundidad crítica de siete secciones hidráulicas (rectangular, trapezoidal, trapezoidal asimétrico, triangular, triangular asimétrico, parabólico y circular). Con el propósito de acelerar y garantizar el nivel de convergencia para cada sección, se obtuvo una función potencial para establecer el valor semiIla en el proceso iterativo; dicho valor se asocia de forma directa a las condiciones preestablecidas del problema hidráulico.
Resultados: La aplicación calcula la profundidad crítica, velocidad crítica, área hidráulica, energía específica, perímetro mojado y el espejo. Los resultados fueron validados contra los obtenidos por medio de la herramienta para análisis de Excel (buscar objetivo) y por el software Hcanales ${ }^{\circledR}$ desarrollado por el ingeniero Máximo Villón Béjar. Finalmente, la aplicación se encuentra disponible de forma gratuita en Play Store (Google), con el nombre de "Flujo Crítico en Canales. Solución Newton Raphson".

Conclusiones: Es posible desarrollar aplicaciones de fácil acceso que cumplan con las condiciones técnicas requeridas para la resolución de situaciones relacionadas con ingeniería.

Palabras clave: código JavaScript, dinámica de fluidos, flujo de agua, programa informático, programa informático didáctico.

\section{INTRODUCTION}

Teaching and learning ways must be in line with technological developments that are experienced today (Contreras, Escobar, \& Tristancho, 2013; Sotelo \&Solarte, 2014). The generation of different learning scenarios leads the student to relate the influence of diverse variables and methodologies in real problems solution (Gómez, Galvis, \& Mariño, 1998). In this sense, applications development for smartphones, becomes an attractive alternative (Gasca, Camargo, \& Medina, 2014), when they are oriented to educational processes (Jonoski et al., 2012). Educational software (ES), are defined as applications or computer programs that facilitate the teaching-learning process (Vidal, Gómez, \& Ruiz, 2010; Drumea, 2012). In the free-flowing hydraulic field, most equations that describe the flow behavior do not resist an analytical development, due mainly, to the nonlinearity of the functions. Which requires numerical methods implementation that solve the hydraulic problem iteratively (Diaz \& Benitez, 1998). In real flow problems it is necessary to use successive approximation methods (Vidal, Gómez, \& Ruiz, 2010). The developed algorithm calculates the critical depth from the numerical method of Newton-Raphson, for 5 successive iterations, based on a preset seed value, which accelerates the convergence.

\section{Critical Flow}

The Froude Number relates the speed, the geometric parameters of the section and the gravitational effects. The critical depth of the flow is defined as the condition for which, the Froude Number (FN) is equal to 1 , where the specific energy is minimal (equation 1). If $\mathrm{FN}<1$, a subcritical flow is established, and if NF $>1$, the flow is supercritical (Figure 1). 


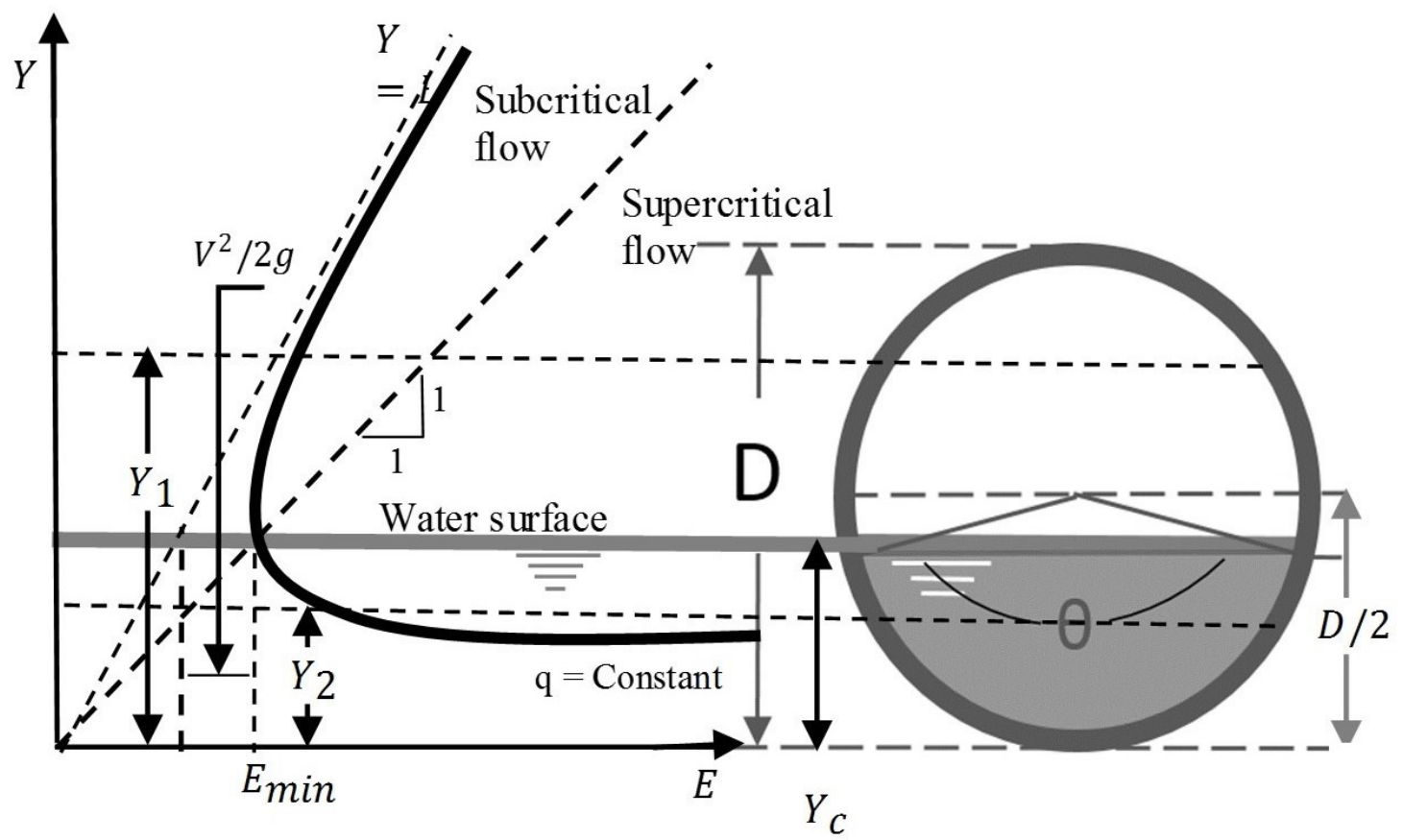

Figure 1. Specific energy diagram

Source: Authors.

Froude:

Newton Raphson Approach:

$$
N F=\frac{V}{\sqrt{g \frac{A}{T}}}
$$

Where: $\mathrm{V}=$ Speed $(\mathrm{m} / \mathrm{s}) ; \mathrm{A}=$ Hydraulic area $\left(\mathrm{m}^{2}\right) ; \mathrm{T}=$ Mirror $(\mathrm{m}) ; \mathrm{g}=$ Gravity $\left(\mathrm{m} / \mathrm{s}^{2}\right)$.

Function:

$$
f\left(Y_{c}\right)=g A^{3} T^{-1}-Q^{2}=0
$$

Where: $Q=$ Flow $\left(\mathrm{m}^{3} / \mathrm{s}\right) ; A=$ Hydraulic area $\left(\mathrm{m}^{2}\right) ; T=\operatorname{Mirror}(\mathrm{m}) ; g=\operatorname{Gravity}\left(\mathrm{m} / \mathrm{s}^{2}\right)$.

Derivative:

$$
f^{\prime}\left(Y_{c}\right)=g\left[-A^{3} T^{-2} \frac{d T}{d Y_{c}}+3 T^{-1} A^{2} \frac{d A}{d Y_{c}}\right]
$$

$$
Y_{c_{n+1}}=Y_{c_{n}}-\frac{f\left(Y_{c_{n}}\right)}{f^{\prime}\left(Y_{c_{n}}\right)}
$$

\section{METHODOLOGY}

For the application development we used iterative and incremental processes (Agile Development), in three specific phases:

- In the first phase, the flow diagram of the iterative process for each of the hydraulic sections (rectangular, trapezoidal, trapezoidal asymmetric, triangular, triangular asymmetric, parabolic and circular) was established.

- In the second phase, the source code (JavaScript) was developed (Lindley, 2013).

- For the last phase, the application was validated by comparing the results against the values obtained by Excel analysis tool (Goal Seek) and by Hcanales ${ }^{\circledR}$ software. 


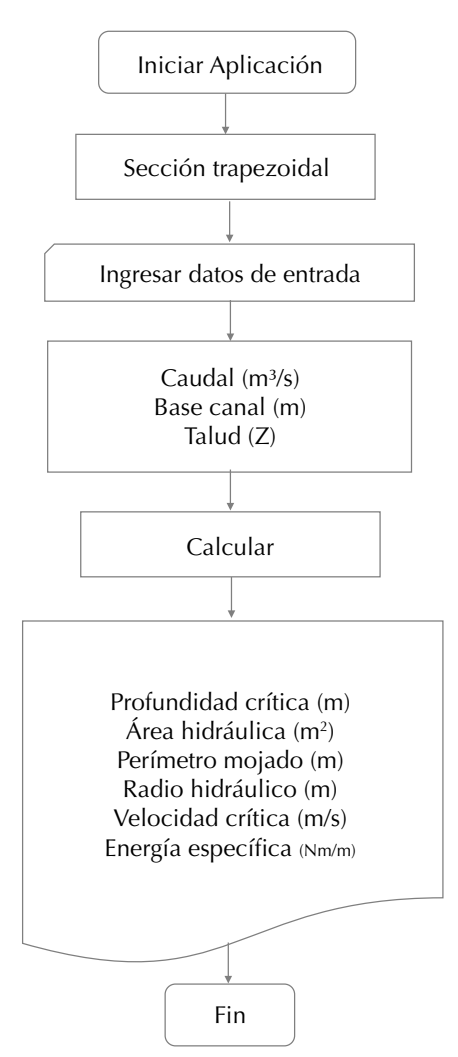

Figure 2. Application flow diagram for the user interface (trapezoidal channel)

Source: Authors.

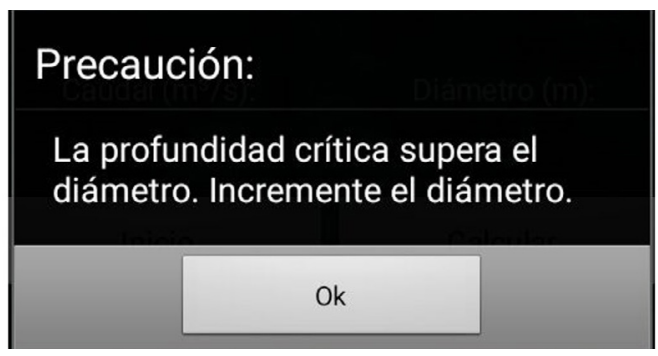

Figure 4. Caution message (circular section) Source: Authors.

The flow chart for the user interface is presented below, which varies according to the selected hydraulic section (Figure 2).

In case of a circular section, the height of critical depth exceeds the diameter value, the application generates a warning, where it is recommended to increase the diameter.
The algorithm shown in Figure 3, was used to program the solution for trapezoidal channel.

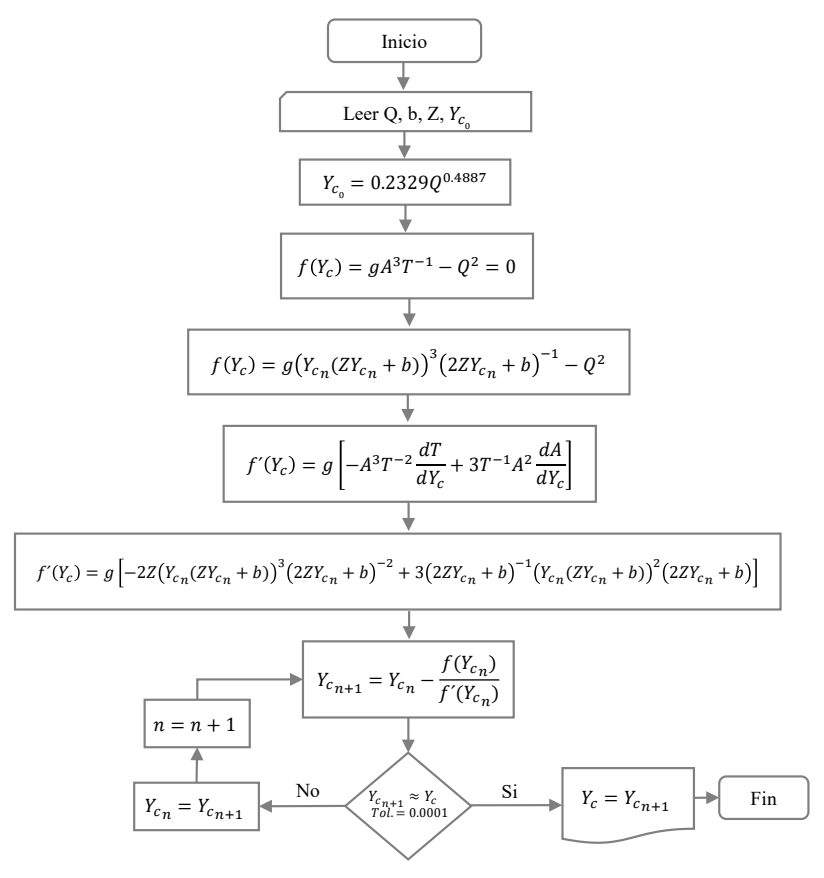

Figure 3. Flow diagram for calculating critical depth (trapezoidal channel)

Source: Authors.

\section{RESULTS}

\section{Trapezoidal Channel}

When you start the application, a menu with seven options of hydraulic sections is displayed (rectangular, trapezoidal, asymmetric trapezoidal, triangular, asymmetric triangular, parabolic and circular). For each section, the algorithm calculates: critical depth, mirror, hydraulic radius, specific energy, flow type, hydraulic area, wet perimeter, critical flow velocity and the Froude number. In order to validate the application, it is proposed to determine the critical depth for a symmetric trapezoidal channel (figure 5), by means of three different methodologies: Newton Raphson, Goal Seek (Excel) and Hcanales ${ }^{\circledR}$. Hydraulic channel conditions are in Table 1. 
Table 1. Input parameters trapezoidal channel

\begin{tabular}{cc}
\hline Flow $\left(\mathrm{m}^{3} / \mathrm{s}\right):$ & 12.25 \\
\hline Base channel $(\mathrm{m}):$ & 2.25 \\
\hline Talud $(\mathrm{Z}):$ & 3.5 \\
\hline
\end{tabular}

Source: Authors.

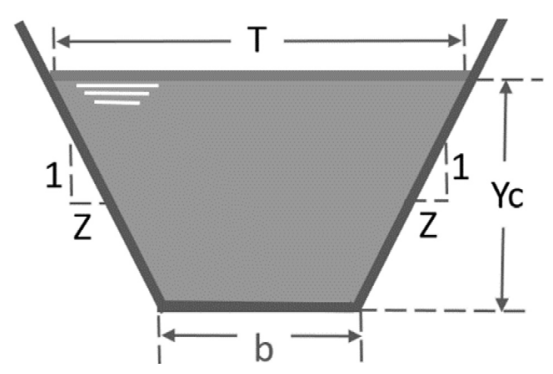

Figure 5. Trapezoidal channel.

Source: Authors.

Hydraulic area:

$$
A=Y_{c}\left(Z Y_{c}+b\right)
$$

Wet perimeter:

$$
P=2 Y_{c} \sqrt{Z^{2}+1}+b
$$

Hydraulic radius:

$$
R=\frac{Y_{C}\left(Z Y_{C}+b\right)}{Y_{C} \sqrt{Z^{2}+1}+b}
$$

Mirror:

$$
T=2 Z Y_{c}+b
$$

Specific Energy:

$$
E e=Y_{c}+\frac{V^{2}}{2 g}
$$

Where: $\mathrm{Q}=$ Flow rate $\left(\mathrm{m}^{3} / \mathrm{s}\right) ; \mathrm{Y} \_\mathrm{c}=$ Critical depth (m).

Mirror derivative:

$$
\frac{d T}{d Y_{c}}=2 Z
$$

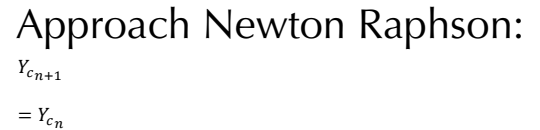

$-\frac{g\left(Y_{c_{n}}\left(Z Y_{c_{n}}+b\right)\right)^{3}\left(2 Z Y_{c_{n}}+b\right)^{-1}-Q^{2}}{g\left[-2 Z\left(Y_{c_{n}}\left(Z Y_{c_{n}}+b\right)\right)^{3}\left(2 Z Y_{c_{n}}+b\right)^{-2}+3\left(2 Z Y_{c_{n}}+b\right)^{-1}\left(Y_{c_{n}}\left(Z Y_{c_{n}}+b\right)\right)^{2}\left(2 Z Y_{c_{n}}+b\right)\right]}$

In the case of symmetric trapezoidal channel, the following function is proposed for seed value:

$$
f\left(Y_{c}\right)=0.2329 Q^{0.4887}
$$

Considering (1), (2) and (3), we obtain:

Derivative hydraulic area:

$$
\frac{d A}{d Y_{c}}=2 Z Y_{c}+b
$$


Tabla 2. Critical depth calculation (Excel, Newton-Raphson), (trapezoidal channel)|

\begin{tabular}{|c|c|c|c|c|c|c|c|c|}
\hline Iter. & Yc n+1 & Area $\left(\mathbf{m}^{2}\right)$ & $\mathbf{T}(\mathbf{m})$ & $\mathrm{dA} / \mathrm{d} \mathrm{Yc}_{\mathrm{c}}$ & $\mathrm{dT} / \mathrm{dYc}$ & $\mathbf{f}\left(Y_{c}\right)$ & $\mathbf{f}^{\prime}(\mathbf{Y c})$ & $\begin{array}{l}\text { Newton } \\
\text { Raphson }\end{array}$ \\
\hline 1 & 0.79239 & 3.98050 & 7.79676 & 7.7968 & 7.000 & -70.7087 & 395.05587 & 0.971379 \\
\hline 2 & 0.97138 & 5.48812 & 9.04965 & 9.0497 & 7.000 & 29.1251 & 747.81227 & 0.932432 \\
\hline 3 & 0.93243 & 5.14097 & 8.77702 & 8.7770 & 7.000 & 1.8024 & 656.70487 & 0.929687 \\
\hline 4 & 0.92969 & 5.11691 & 8.75781 & 8.7578 & 7.000 & 0.0084 & 650.60865 & 0.929674 \\
\hline 5 & 0.92967 & 5.11680 & 8.75772 & 8.7577 & 7.000 & 0.0000 & 650.58016 & 0.9297 \\
\hline
\end{tabular}

Source: Authors.

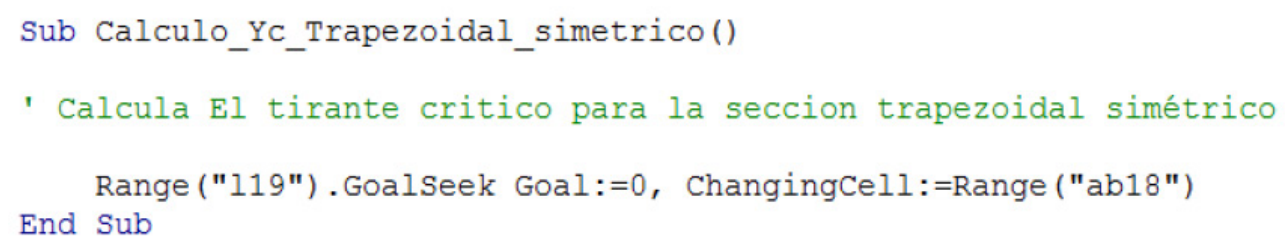

Figure 6. Visual Code (Excel). Iteration for Yc calculation (symmetric trapezoidal channel) Fuente: Authors.

The results obtained from these three methodologies, generate the same value for the critical depth, under the initial conditions established.

Table 3. Comparison of Yc results (trapezoidal channel)

\begin{tabular}{ccccc}
\hline & \multicolumn{2}{c}{ Excel } & & \\
\cline { 2 - 3 } Parameter & Goal Seek & $\begin{array}{c}\text { Newton } \\
\text { Raphson }\end{array}$ & HCanales (Software) & Application (JavaScript) \\
\hline Critical depth $(\mathrm{m}):$ & 0.9297 & 0.9297 & 0.9297 & 0.9297 \\
\hline Mirror $(\mathrm{m}):$ & 8.7577 & 8.7577 & 8.7577 & 8.7577 \\
\hline Hydraulic radius $(\mathrm{m}):$ & 0.5674 & 0.5674 & 0.5674 & 0.5674 \\
\hline Specific energy $(\mathrm{Nm} / \mathrm{N}):$ & 1.2218 & 1.2218 & 1.2218 & 1.2218 \\
\hline Hydraulic area $\left(\mathrm{m}^{2}\right):$ & 5.1168 & 5.1168 & 5.1168 & 5.1168 \\
\hline Perimeter $(\mathrm{m}):$ & 9.0181 & 9.0181 & 9.0181 & 2.0181 \\
\hline Critical speed $(\mathrm{m} / \mathrm{s}):$ & 2.3941 & 2.3941 & 2.3941 & 1.0000 \\
\hline Froude number: & 1.0000 & 1.0000 & 1.0000 & 2.3941 \\
\hline
\end{tabular}

Source: Authors. 

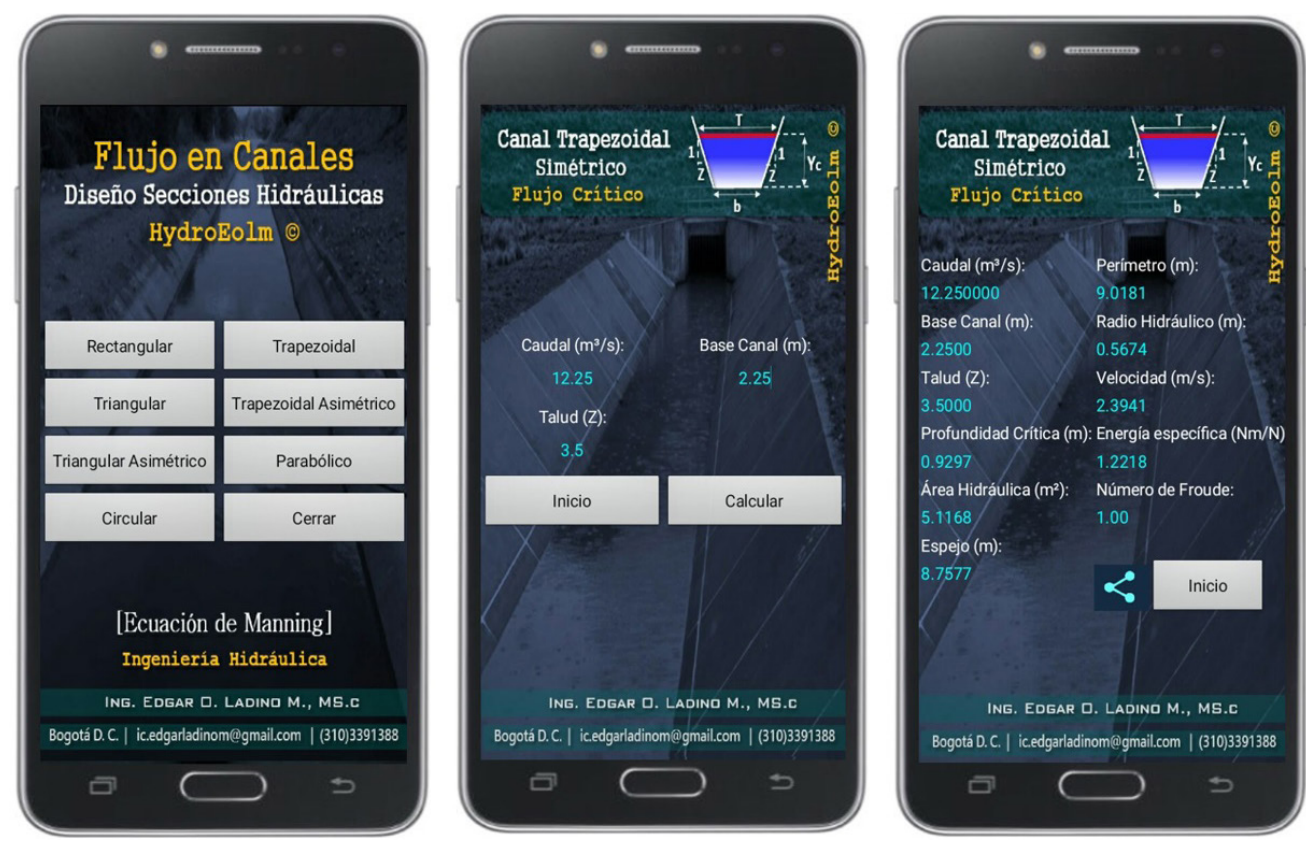

Figure 7. Trapezoidal channel, (Application solution for critical depth) Fuente: Authors.

\section{Asymmetric Trapezoidal Channel}

Considering (1), and with the purpose of verifying the capacity of the application for critical depth calculation, we proposed an asymmetric trapezoidal section, with these hydraulic conditions:

Table 4. Input parameters asymmetric trapezoidal channel

\begin{tabular}{cc}
\hline Flow $\left(\mathrm{m}^{3} / \mathrm{s}\right):$ & 1.75 \\
\hline Base channel $(\mathrm{m}):$ & 1.2 \\
\hline Talud $(\mathrm{ZA}):$ & 5 \\
\hline Talud $(\mathrm{ZB}):$ & 2 \\
\hline
\end{tabular}

Source: Authors.

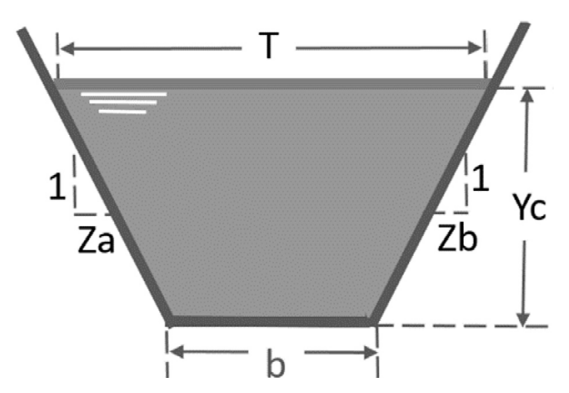

Figure 5. Trapezoidal channel.

Source: Authors. 
Hydraulic area:

$$
A=0.5\left(Z_{a} Y_{c}^{2}+2 b Y_{c}+Z_{b} Y_{c}^{2}\right)
$$

Wet perimeter:

$$
P=Y_{c} \sqrt{Z_{a}^{2}+1}+b+Y_{c} \sqrt{Z_{b}^{2}+1}
$$

Hydraulic radius:

$$
R=\frac{0.5\left(Z_{a} Y_{c}^{2}+2 b Y_{c}+Z_{b} Y_{c}^{2}\right)}{Y_{c} \sqrt{Z_{a}^{2}+1}+b+Y_{c} \sqrt{Z_{b}^{2}+1}}
$$

Mirror:

$$
T=Z_{a} Y_{c}+b+Z_{b} Y_{c}
$$

Hydraulic area derivative:

$$
\frac{d A}{d Y_{c}}=Z_{a} Y_{c}+b+Z_{b} Y_{c}
$$

Mirror derivative:

$$
\frac{d T}{d Y_{c}}=Z_{a}+Z_{b}
$$

From equations (2), (3) and (4), we can conclude that:

Newton Raphson Approach:

$$
f\left(Y_{c_{n}}\right)=g\left(0.5\left(Z_{a} Y_{c_{n}}^{2}+2 b Y_{c_{n}}+Z_{b} Y_{c}^{2}\right)\right)^{3}\left(Z_{a} Y_{c_{n}}+b+Z_{b} Y_{c_{n}}\right)^{-1}-Q^{2}
$$

$$
\begin{aligned}
f^{\prime}\left(Y_{c_{n}}\right)=g[ & -\left(0.5\left(Z_{a} Y_{c_{n}}^{2}+2 b Y_{c_{n}}+Z_{b} Y_{Y_{n}}^{2}\right)\right)^{3}\left(Z_{a} Y_{c_{n}}+b+Z_{b} Y_{c_{n}}\right)^{-2}\left(Z_{a}+Z_{b}\right) \\
& +3\left(Z_{a} Y_{c_{n}}+b+Z_{b} Y_{c_{n}}\right)^{-1}\left(0.5\left(Z_{a} Y_{c_{n}}^{2}+2 b Y_{c_{n}}+Z_{b} Y_{c_{n}}\right)\right)^{2}\left(Z_{a} Y_{c}+b\right. \\
& \left.\left.+Z_{b} Y_{c}\right)\right]
\end{aligned}
$$

For the asymmetric trapezoidal channel, a potential function was implemented: critical depth vs. flow, for the initial value (seed value). This function accelerates the numerical method convergence. The visual code used in Excel is shown in Figure 9.

$$
f\left(Y_{c}\right)=0.2379 Q^{0.5045}
$$

Table 5. Critical depth calculation (Excel, Newton-Raphson) (Asymmetric trapezoidal channel)

\begin{tabular}{ccccccccc}
\hline Iter. & $\mathbf{Y} \mathbf{n}+\mathbf{1}$ & Area $\left(\mathbf{m}^{\mathbf{2}}\right)$ & $\mathbf{T}(\mathbf{m})$ & $\mathbf{d A} / \mathbf{d} \mathbf{c}$ & $\mathbf{d T / d Y c}$ & $\mathbf{f}(\mathbf{Y c})$ & $\mathbf{f}^{\prime}(\mathbf{Y c})$ & $\begin{array}{c}\text { Newton } \\
\text { Raphson }\end{array}$ \\
\hline $\mathbf{1}$ & 0.31551 & 0.7270 & 3.4085 & 3.4085 & 7.000 & -1.9566 & 13.28386 & 0.462796 \\
\hline $\mathbf{2}$ & 0.46280 & 1.3049 & 4.4395 & 4.4396 & 7.000 & 1.8482 & 42.37605 & 0.419181 \\
\hline $\mathbf{3}$ & 0.41918 & 1.1180 & 4.1342 & 4.1343 & 7.000 & 0.2535 & 31.17162 & 0.411050 \\
\hline $\mathbf{4}$ & 0.41105 & 1.0846 & 4.0773 & 4.0773 & 7.000 & 0.0075 & 29.35139 & 0.410796 \\
\hline $\mathbf{5}$ & 0.41080 & 1.0835 & 4.0755 & 4.0756 & 7.000 & 0.0000 & 29.29582 & 0.4108 \\
\hline
\end{tabular}

Source: Authors.

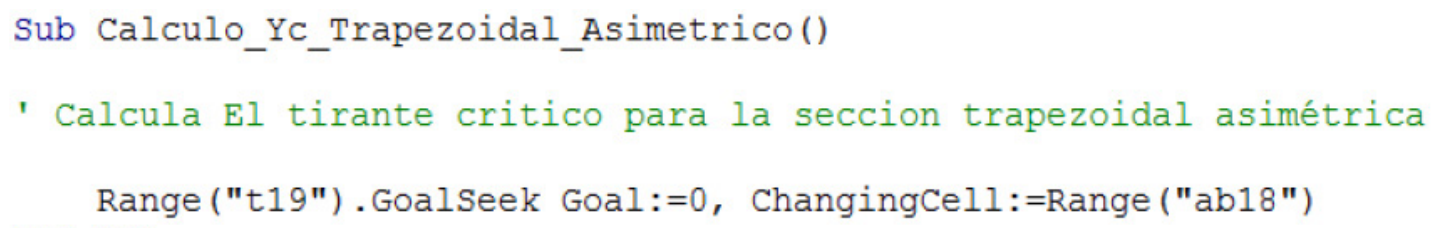

Figure 9. Visual code (Excel). Iteration for Yc calculation. (Asymmetric trapezoidal channel) Fuente: Authors. 
Table 6. Comparison of $Y_{c}$ results (Asymmetric trapezoidal channel)

\begin{tabular}{cccc}
\hline & \multicolumn{2}{c}{ Excel } & \\
\cline { 2 - 3 } Parameter & Goal Seek & $\begin{array}{c}\text { Newton } \\
\text { Raphson }\end{array}$ & HCanales (Software) \\
\hline Critical depth $(\mathrm{m}):$ & 0.4108 & 0.4108 & 0.4108 \\
\hline Mirror $(\mathrm{m}):$ & 4.0756 & 4.0756 & 4.0756 \\
\hline Hydraulic radius $(\mathrm{m}):$ & 0.2572 & 0.2572 & 0.2572 \\
\hline Specific energy $(\mathrm{Nm} / \mathrm{N}):$ & 0.5437 & 0.5437 & 0.5437 \\
\hline Hydraulic area $\left(\mathrm{m}^{2}\right):$ & 1.0836 & 1.0836 & 1.0836 \\
\hline Perimeter $(\mathrm{m}):$ & 4.2132 & 4.2132 & 4.2132 \\
\hline Critical speed $(\mathrm{m} / \mathrm{s}):$ & 1.6150 & 1.6150 & 1.6150 \\
\hline Froude number: & 1.0000 & 1.0000 & 1.0000 \\
\hline
\end{tabular}

Source: Authors.
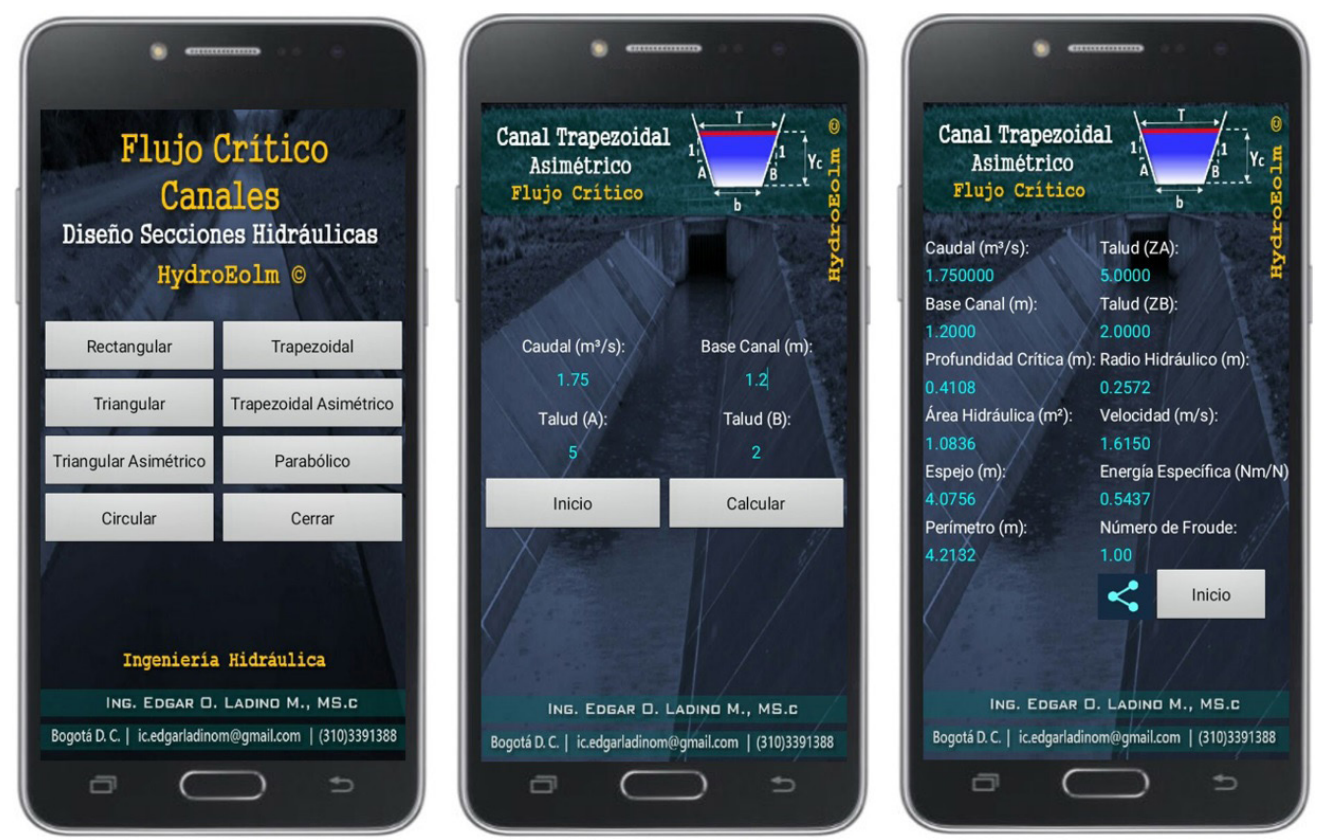

Figure 10. Asymmetric trapezoidal channel (Application critical depth solution) Fuente: Authors. 


\section{Circular Channel}

To evaluate the Application results, we present the following hydraulic problem:

Table 7. Input parameters circular channel

\begin{tabular}{cc}
\hline Flow $\left(\mathrm{m}^{3} / \mathrm{s}\right):$ & 0.145 \\
\hline Diameter $(\mathrm{m}):$ & 0.4 \\
\hline
\end{tabular}

Source: Authors.

Figure 11. Circular channel Source: Authors.

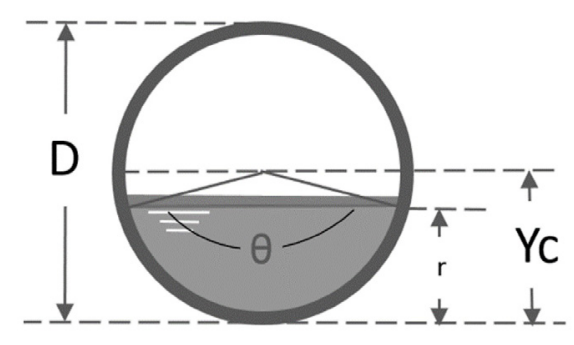

\section{Central Angle}

$$
\theta=2 \operatorname{acos}\left[1-\frac{2 Y_{c}}{D}\right]
$$

Hydraulic area:

$$
A=\frac{D^{2}}{8}(\theta-\sin \theta)
$$

Wet perimeter:

$$
P=\frac{\theta D}{2}
$$

Hydraulic radius:

$$
R=\frac{D(\theta-\sin \theta)}{4 \theta}
$$

Mirror:

$$
T=D \sin (\theta / 2)
$$

Hydraulic area derivative:

$$
E e=Y_{c}+\frac{V^{2}}{2 g}
$$

Newton Raphson Approach:

Function:

$$
f(\theta)=\left[\sin \left(\frac{\theta}{2}\right)\right]^{\frac{1}{3}}[\theta-\sin \theta]^{-1}-\frac{A_{\text {full }}}{2 \pi\left[\frac{Q^{2} D}{g}\right]^{\frac{1}{3}}}=0
$$

Derivative:

$$
f^{\prime}(\theta)=\left\lfloor-\left[\sin \left(\frac{\theta}{2}\right)\right]^{\frac{1}{3}}[\theta-\sin \theta]^{-2}[1-\cos \theta]\right.
$$

\section{Where:}

$$
\left.+\frac{1}{6}[\theta-\sin \theta]^{-1}\left[\sin \left(\frac{\theta}{2}\right)\right]^{-\frac{2}{3}}\left[\cos \left(\frac{\theta}{2}\right)\right]\right] \frac{d \theta}{d Y_{c}}
$$

$$
\begin{aligned}
& \frac{d \theta}{d Y_{c}}=\frac{4 / D}{\sqrt{1-\left[1-\frac{2 Y_{n}}{D}\right]^{2}}} \\
& \theta_{n+1}=\theta_{n}-\frac{f\left(\theta_{n}\right)}{f^{\prime}\left(\theta_{n}\right)} \\
& =\theta_{n}
\end{aligned}
$$

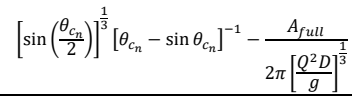

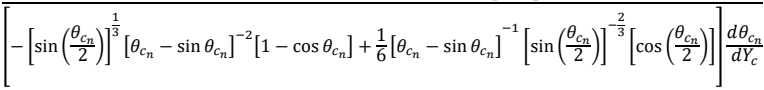


Table 8. Yc solution (Excel - Newton-Raphson) (circular channel)

\begin{tabular}{|c|c|c|c|c|c|c|c|c|}
\hline Iter. & Yc n+1 & Area $\left(\mathbf{m}^{2}\right)$ & $\mathbf{T}(\mathbf{m})$ & $\mathrm{dA} / \mathrm{dYc}$ & $d T / d Y c$ & $f(Y c)$ & $f^{\prime}(Y c)$ & $\begin{array}{l}\text { Newton } \\
\text { Raphson }\end{array}$ \\
\hline 1 & 0.21035 & 3.24511 & 10.01341 & 0.08798 & -1.8064238 & 0.259050819 & 13.28386 & 0.462796 \\
\hline 2 & 0.25905 & 3.74104 & 10.46661 & 0.01824 & -1.07705017 & 0.275984611 & 42.37605 & 0.419181 \\
\hline 3 & 0.27598 & 3.92102 & 10.81060 & 0.00019 & -0.92107472 & 0.276189028 & 31.17162 & 0.411050 \\
\hline 4 & 0.27619 & 3.92323 & 10.81552 & -0.00002 & -0.91939261 & 0.276171857 & 29.35139 & 0.410796 \\
\hline 5 & 0.27617 & 3.92304 & 10.81510 & 0.00000 & -0.91953374 & 0.2762 & 29.29582 & 0.4108 \\
\hline
\end{tabular}

Source: Authors.

\section{Sub Calculo_Yc_Circular()}

' Calcula El tirante critico para la seccion circular

\section{Range ("s29").Goalseek Goal:=0, ChangingCell:=Range ("ad29") End Sub}

Figure 12. Visual code (Excel). Iteration for Yc calculation

Fuente: Authors.

Figure 13. was constructed from the section factor for critical flow.

$$
A^{3} T^{-1}=g^{-1} Q^{2}
$$

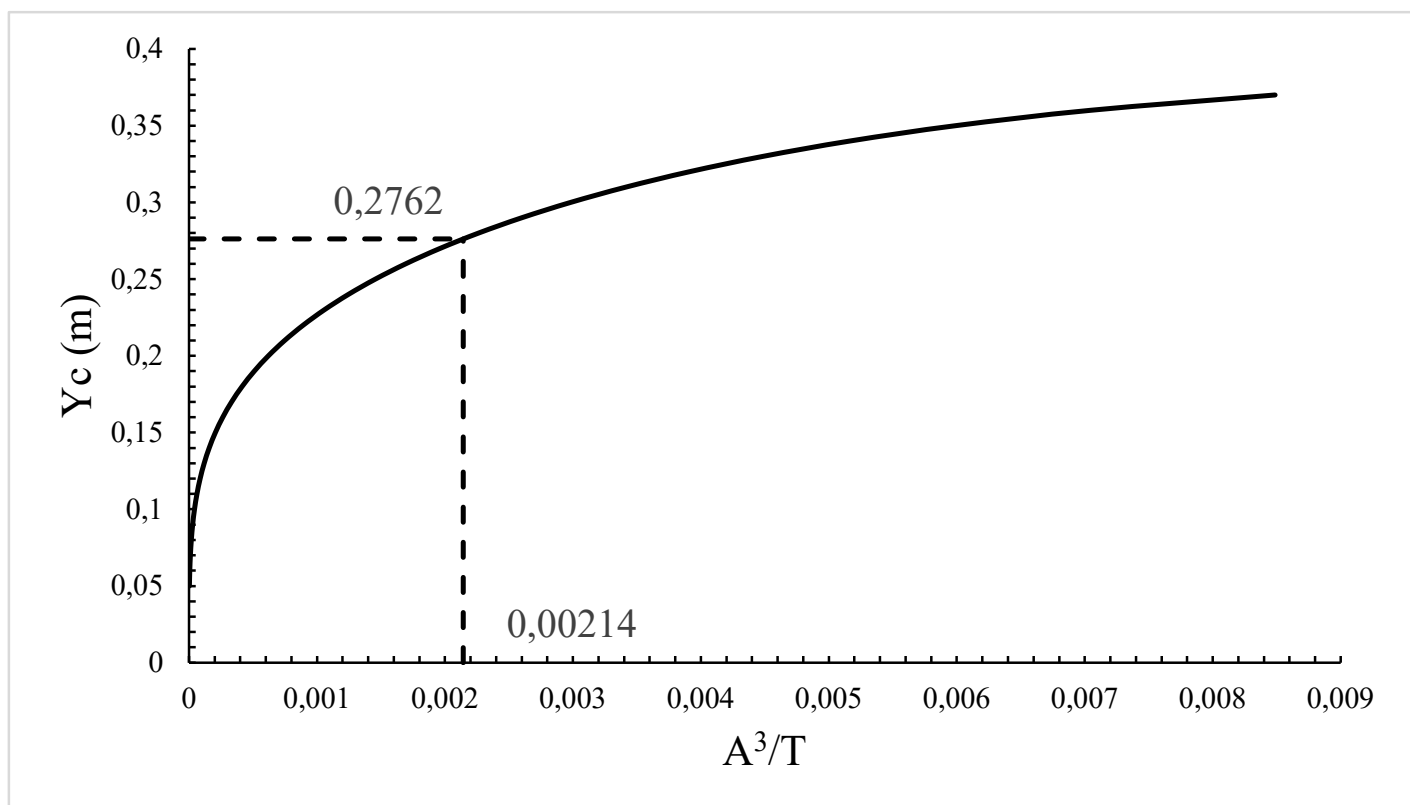

Figure 13. Circular pipe (graphic solution) Fuente: Authors. 

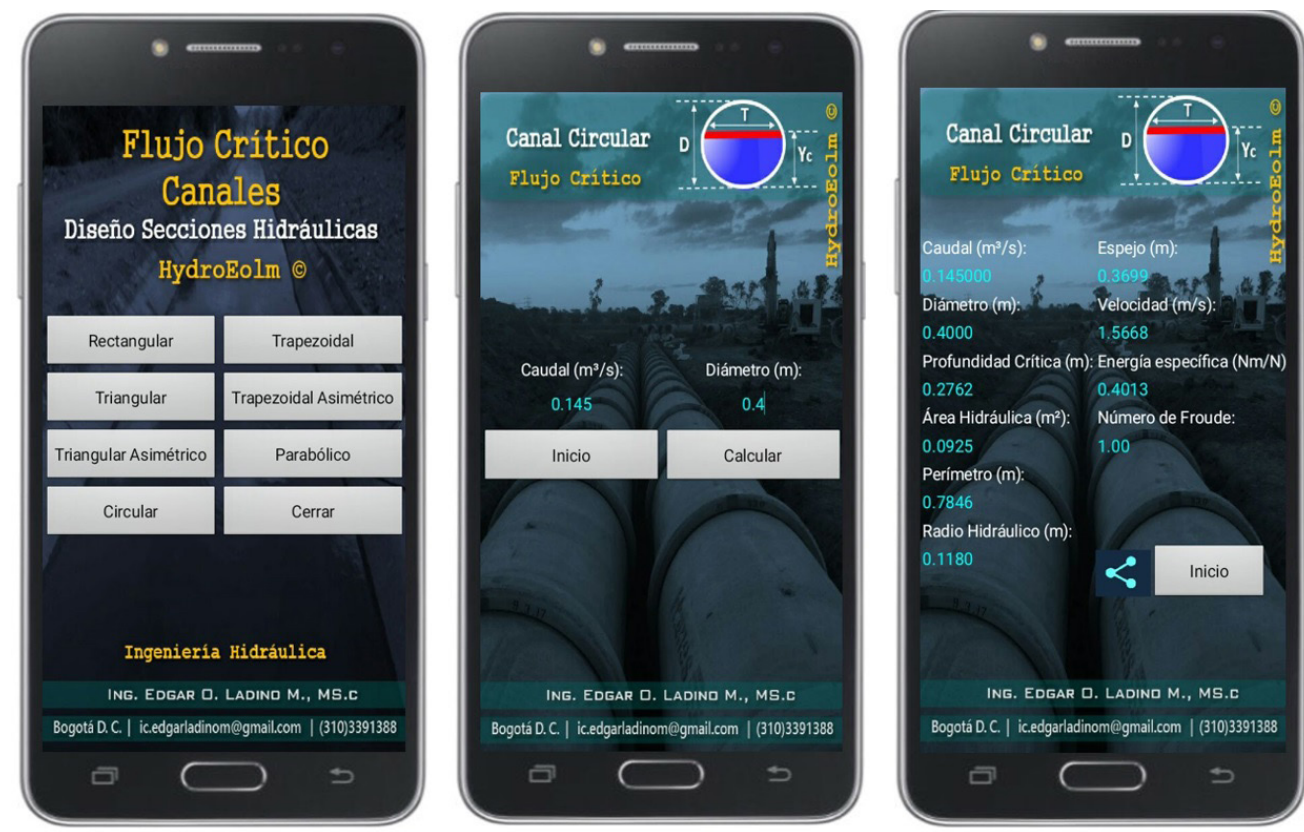

Figure 14. Circular channel, (Application critical depth calculation) Source: Authors.

Table 9. Comparison of $Y_{C}$ results (circular channel)

\begin{tabular}{ccccc}
\hline \multirow{2}{*}{ Parameter } & Goal Seek & $\begin{array}{c}\text { Newton } \\
\text { Raphson }\end{array}$ & HCanales (Software) & Application (JavaScript) \\
\cline { 2 - 3 } Critical depth $(\mathrm{m}):$ & 0.2762 & 0.2762 & 0.2762 & 0.2762 \\
\hline Mirror $(\mathrm{m}):$ & 0.3699 & 0.3699 & 0.3699 & 0.3699 \\
\hline Hydraulic radius $(\mathrm{m}):$ & 0.1180 & 0.1180 & 0.1180 & 0.1180 \\
\hline Specific energy $(\mathrm{Nm} / \mathrm{N}):$ & 0.4013 & 0.4013 & 0.4013 & 0.4013 \\
\hline Hydraulic area $\left(\mathrm{m}^{2}\right):$ & 0.0925 & 0.0925 & 0.0925 & 0.0925 \\
\hline Perimeter $(\mathrm{m}):$ & 0.7846 & 0.7846 & 0.7846 & 0.7846 \\
\hline Critical speed $(\mathrm{m} / \mathrm{s}):$ & 1.5668 & 1.5668 & 1.5668 & 1.5668 \\
\hline Froude number: & 1.0000 & 1.0000 & 1.0000 & 1.0000 \\
\hline
\end{tabular}

Source: Authors. 
Finally, the application was submitted to some exercises development proposed in different Hydraulics books. In order to validate the algorithm developed, the results are presented in tables 10, 11 and 12. Similarly, the results calculated by the Application are shown in figures 15, 16 and 17.

Table 10. Results comparison Exercise 3.5, Hydraulics of channels

\begin{tabular}{|c|c|c|c|c|c|c|}
\hline \multirow[t]{2}{*}{ Book } & \multirow[t]{2}{*}{ Exercise } & \multicolumn{2}{|c|}{$\begin{array}{c}\text { Input } \\
\text { parameters }\end{array}$} & \multirow{2}{*}{$\begin{array}{c}\begin{array}{c}\text { Output } \\
\text { parameters }\end{array} \\
\begin{array}{c}\text { Critical depth } \\
(\mathrm{m}):\end{array} \\
\end{array}$} & \multirow{2}{*}{$\begin{array}{c}\text { Book } \\
1.1710\end{array}$} & \multirow{2}{*}{$\begin{array}{c}\text { Application } \\
1.1710\end{array}$} \\
\hline & & $\begin{array}{l}\text { Flow } \\
\left(\mathrm{m}^{3} / \mathrm{s}\right):\end{array}$ & 20 & & & \\
\hline \multirow[t]{2}{*}{$\begin{array}{c}\text { Channel } \\
\text { hy-draulics } \\
\text { (Sotelo, 2002) }\end{array}$} & $\begin{array}{c}3.5 \\
\text { (Pg. 195) }\end{array}$ & $\begin{array}{c}\text { Base } \\
\text { chan- } \\
\text { nel }(\mathrm{m}) \text { : }\end{array}$ & 4 & $\begin{array}{c}\text { Critical velocity } \\
\qquad(\mathrm{m} / \mathrm{s}):\end{array}$ & 2.967 & 2.9668 \\
\hline & & $\begin{array}{l}\text { Talud } \\
\text { (Z): }\end{array}$ & 1.5 & $\begin{array}{c}\text { Specific energy } \\
(\mathrm{Nm} / \mathrm{m}):\end{array}$ & 1.62 & 1.6197 \\
\hline
\end{tabular}

Source: Authors.

Table 11. Results comparison Exercise 4.2, Hydraulics of open channels

\begin{tabular}{|c|c|c|c|c|c|c|}
\hline Book & Exercise & $\begin{array}{r}\text { I } \\
\text { parc }\end{array}$ & $\begin{array}{l}\text { put } \\
\text { meters }\end{array}$ & $\begin{array}{c}\text { Output } \\
\text { parameters }\end{array}$ & Book & Application \\
\hline \multirow{3}{*}{$\begin{array}{l}\text { Hydraulics of } \\
\text { open channels } \\
\text { (Chow, 1994) }\end{array}$} & \multirow{3}{*}{$\begin{array}{c}4.2 \\
\text { (Pg. 68) }\end{array}$} & $\begin{array}{l}\text { Flow } \\
\left(\mathrm{m}^{3} / \mathrm{s}\right) \text { : }\end{array}$ & 11.32674 & $\begin{array}{l}\text { Critical depth } \\
(\mathrm{m}):\end{array}$ & 0.6553 & 0.6547 \\
\hline & & $\begin{array}{c}\text { Base } \\
\text { chan- } \\
\text { nel }(\mathrm{m}) \text { : }\end{array}$ & 6.0960 & $\begin{array}{l}\text { Critical velocity } \\
\qquad(\mathrm{m} / \mathrm{s}):\end{array}$ & 2.3336 & 2.3362 \\
\hline & & $\begin{array}{l}\text { Talud } \\
\text { (Z): }\end{array}$ & 2 & $\begin{array}{c}\text { Specific energy } \\
(\mathrm{Nm} / \mathrm{m}):\end{array}$ & 0.9329 & 0.9329 \\
\hline
\end{tabular}

Source: Authors.

Table 12. Results comparison Exercise 4.3, Hydraulics of open channels

\begin{tabular}{|c|c|c|c|c|c|c|}
\hline Book & Exercise & par & $\begin{array}{l}\text { put } \\
\text { neters }\end{array}$ & $\begin{array}{c}\text { Output } \\
\text { parameters }\end{array}$ & Book & Application \\
\hline \multirow{3}{*}{$\begin{array}{l}\text { Hydraulics of } \\
\text { open channels } \\
\text { (Chow, 1994) }\end{array}$} & \multirow{3}{*}{$\begin{array}{c}4.3 \\
\text { (Pg. 68) }\end{array}$} & $\begin{array}{l}\text { Flow } \\
\left(\mathrm{m}^{3} / \mathrm{s}\right) \text { : }\end{array}$ & 0.566334 & $\begin{array}{c}\text { Critical depth } \\
(\mathrm{m}) \text { : }\end{array}$ & 0.4389 & 0.4374 \\
\hline & & $\begin{array}{c}\text { Base } \\
\text { chan- } \\
\text { nel }(m) \text { : }\end{array}$ & 0.9144 & $\begin{array}{l}\text { Critical velocity } \\
\qquad(\mathrm{m} / \mathrm{s}):\end{array}$ & 1.8174 & 1.8253 \\
\hline & & & & $\begin{array}{c}\text { Specific energy } \\
(\mathrm{Nm} / \mathrm{m}):\end{array}$ & 0.6072 & 0.6072 \\
\hline
\end{tabular}

Source: Authors. 

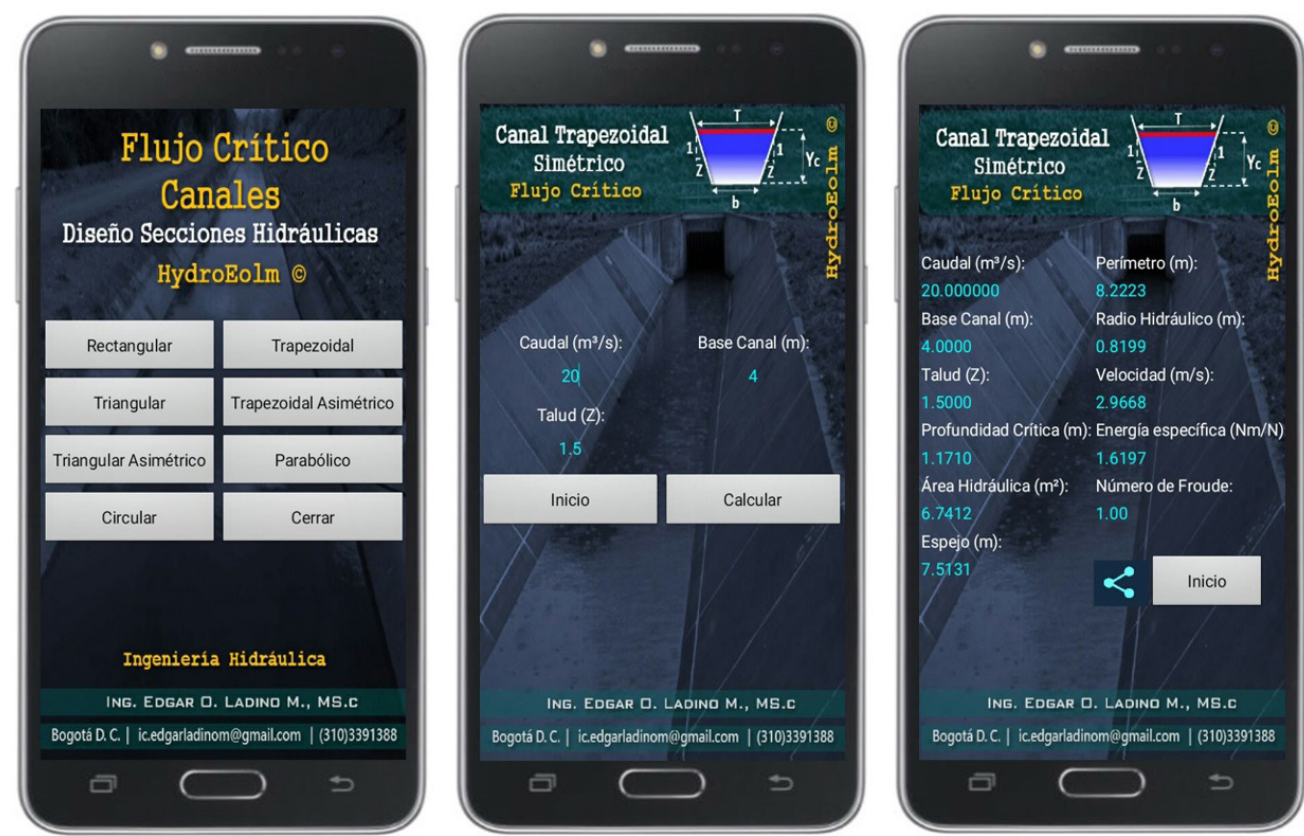

Figure 15. Exercise 3.5, Hydraulics of channels, Gilberto Sotelo (Application Critical depth calculation) Source: Authors.
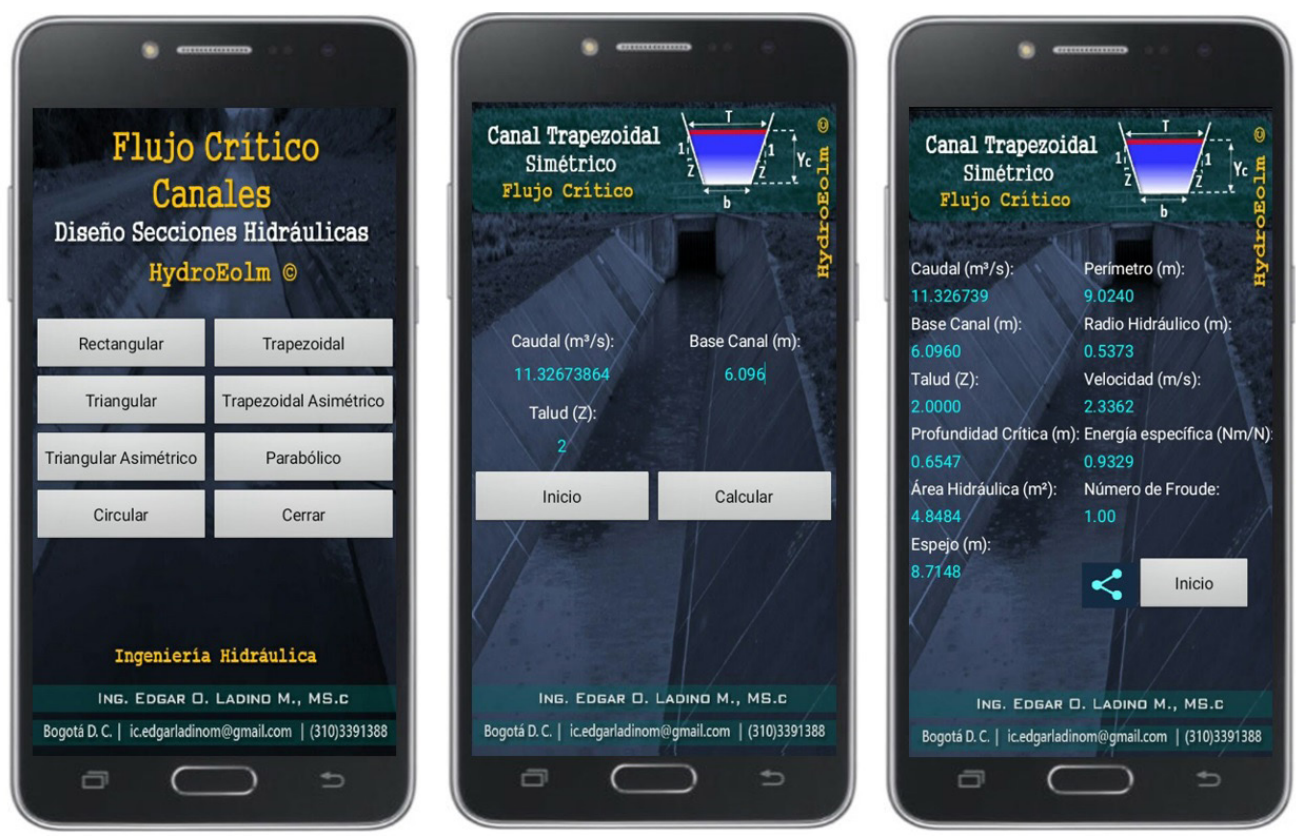

Figure 16. Exercise 4.2, Hydraulics of open channels, Ven Te Chow (Application Critical depth calculation) Source: Authors. 

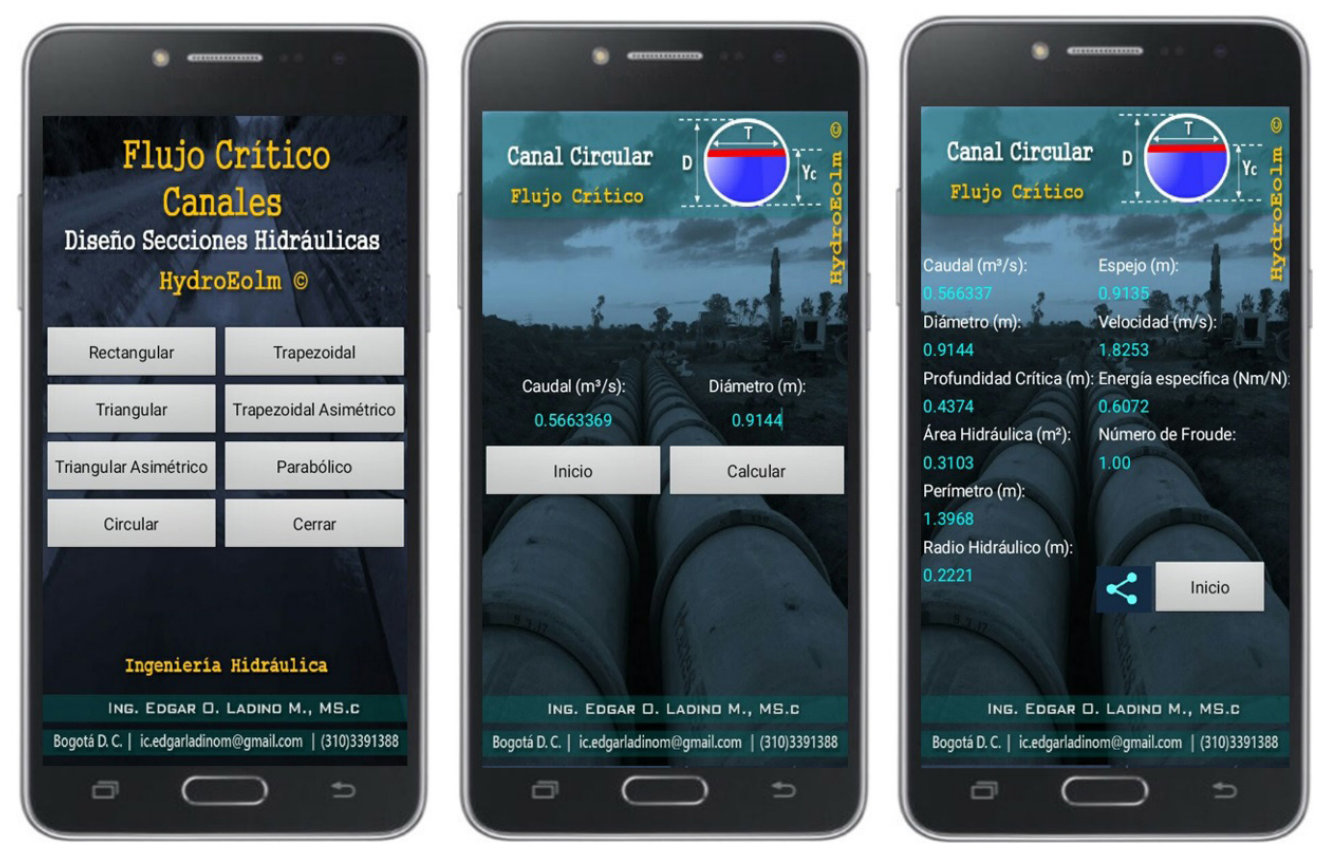

Figure 17. Exercise 4.3, Hydraulics of open channels, Ven Te Chow (Application Critical depth calculation) Source: Authors.

\section{CONCLUSIONS}

The algorithm developed for the application "Diseño de Canales Hidráulicos HydroEolm" under JavaScript programming language avoids the use of calculation curves for critical depth. The results showed that the application effectively calculates the critical depth, critical speed, hydraulic area, specific energy, wet perimeter and the mirror.

In the application development, the results were compared with different exercises proposed in Hydraulics books, showing optimal outputs. Likewise, about 95 hydraulic exercises were performed, which were validated by comparing the application results against results obtained from three different methodologies (Goal Seek, Newton-Rapson, Hcanales ${ }^{\circledR}$.

Finally, the development of algorithms for operating systems compatible with iOS and Windows is recommended, which expands the coverage of the application to a greater number of users. This application has been downloaded in Colombia, Bolivia, Peru, Costa Rica and Algeria.

\section{REFERENCES}

Chow, V. T. (1994). Hidráulica de Canales Abiertos. Bogotá D.C.: McGraw-Hill.

Contreras, L.E., Escobar, I., \&Tristancho,J.A. (2013). Estrategias educativas para el uso de las TIC en educación superior. Tecnura, 17, 161-173. doi:https://doi.org/10.14483/22487638.7246

Diaz, J., \& Benitez, F. (1998). Introducción a los métodos numéricos para la resolución de ecuaciones. Cádiz: Universidad de Cádiz.

Drumea, A. (2012). Education in development of electronic modules using free and open source software tools. Hidraulica, (3/4), 54-60. 
Gasca, M. C., Camargo, L. L., \& Medina, B. (2014). Metodología para el desarrollo de aplicaciones móviles. Tecnura, 18(40), 20-35.

Gómez, R., Galvis, A., \& Mariño, O. (1998). Ingeniería de software educativo con modelaje orientado por objetos: un medio para desarrollar micromundos interactivos. Informática Educativa Uniandes Lidie, 11(1), 9-30.

Jonoski, A., Alfonso, L., Almoradie, A., Popescu, I., Van Andel, S., \& Vojinovik, Z. (2012). Mobile phone applications in the water domain. Environmental Engineering and Management Journal, 11, 919-930.

Lindley, C. (2013). JavaScript Enligthment. Sebastopol: O'Reilly Media Inc.

Sotelo, F., \& Solarte, M. F. (2014). Incorporación de recursos web como servicios de e-learning al sistema de gestión de aprendizaje. LRN: una revisión. Tecnura, 18(39), 165-180. doi:https://doi.org/10.14483/ udistrital.jour.tecnura.2014.1.a12

Sotelo, G. (2002). Hidráulica de Canales. México: Facultad de Ingeniería de la UNAM.

Vidal, M., Gómez, F., \& Ruiz, A. (2010). Software Educativos. Educación Médica Superior, 24(1). Retrieved from http:// scielo.sld.cu/scielo.php?script=sci_arttext\&pid=S0864-21412010000100012

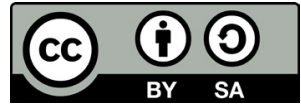

